JOURNAL OF APPLIED CRYSTALLOGRAPHY

ISSN 1600-5767

Received 11 November 2019

Accepted 13 December 2019

Edited by S. Boutet, SLAC National Accelerator Laboratory, Menlo Park, USA

Keywords: X-ray crystallography; microcrystals; data assembly; radiation damage; multi-crystal; Python.
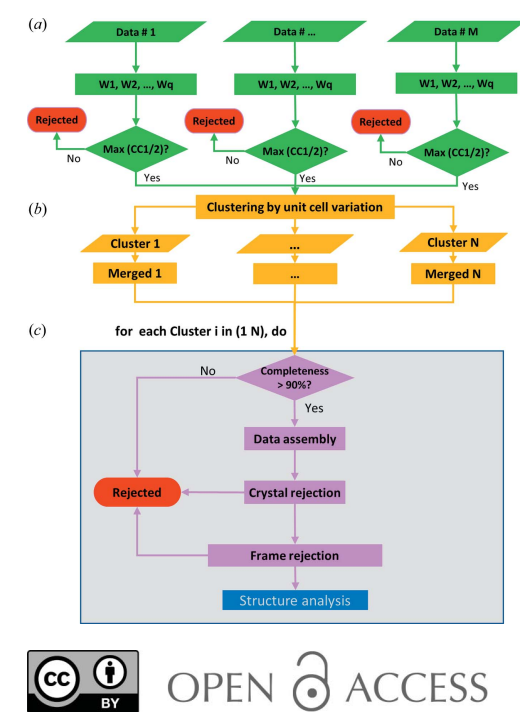

\section{PyMDA: microcrystal data assembly using Python}

\author{
Lina Takemaru, ${ }^{a}$ Gongrui Guo, ${ }^{\text {a,b }}$ Ping Zhu, ${ }^{a}$ Wayne A. Hendrickson, ${ }^{c, d}$ Sean \\ McSweeney ${ }^{b}$ and Qun Liu ${ }^{a, b *}$
}

\begin{abstract}
a Biology Department, Brookhaven National Laboratory, Upton, NY 11973, USA, ${ }^{\mathbf{b}}$ Photon Sciences Division, NSLS-II, Brookhaven National Laboratory, Upton, NY 11973, USA, 'Department of Biochemistry and Molecular Biophysics, Columbia University, New York, NY 10032, USA, and ${ }^{\mathbf{d}}$ Department of Physiology and Cellular Biophysics, Columbia University, New York, NY 10032, USA. *Correspondence e-mail: qunliu@bnl.gov
\end{abstract}

The recent developments at microdiffraction X-ray beamlines are making microcrystals of macromolecules appealing subjects for routine structural analysis. Microcrystal diffraction data collected at synchrotron microdiffraction beamlines may be radiation damaged with incomplete data per microcrystal and with unit-cell variations. A multi-stage data assembly method has previously been designed for microcrystal synchrotron crystallography. Here the strategy has been implemented as a Python program for microcrystal data assembly $(P y M D A)$. PyMDA optimizes microcrystal data quality including weak anomalous signals through iterative crystal and frame rejections. Beyond microcrystals, PyMDA may be applicable for assembling data sets from larger crystals for improved data quality.

\section{Introduction}

Biomolecular X-ray crystallography has enabled the understanding of biological complexity at the atomic and molecular level. The optimization of crystals to suitable sizes is a bottleneck in biomolecular crystallography. Thus, for many difficult-to-optimize samples such as membrane proteins and eukaryotic proteins, using their microcrystals for structural analysis is appealing. With the recent developments at synchrotron microdiffraction beamlines (Flot et al., 2010; Evans et al., 2011; Diederichs \& Wang, 2017; Yamamoto et al., 2017) and X-ray free-electron lasers (XFELs) (Schlichting, 2015; Spence, 2017), microcrystals produce high-resolution diffraction patterns. Compared with XFELs which produce only one diffraction pattern for every microcrystal, synchrotron microdiffraction beamlines are optimized for collection of a small wedge of rotation data from each microcrystal, thus greatly improving data quality from microcrystals (Smith et al., 2012; Fuchs et al., 2016; Yamamoto et al., 2017). With the implementation of new data collection methods, microcrystal data acquisition at synchrotrons is now routine and maturing (Gati et al., 2014; Coquelle et al., 2015; Zander et al., 2015; Diederichs \& Wang, 2017; Owen et al., 2017; Sanishvili \& Fischetti, 2017; Gao et al., 2018; Huang et al., 2018; Basu et al., 2019; Cianci et al., 2019; Dauter, 2019; Guo et al., 2019).

Because of radiation damage, the lifetime of a microcrystal does not allow for the collection of complete rotation data even under cryogenic conditions (Holton \& Frankel, 2010; Zeldin et al., 2013; Garman \& Weik, 2017; Warren et al., 2019). In general, only a few degrees of rotation data may be collected from a single microcrystal of a few micrometres. The problem of how to assemble data from radiation-damaged and incomplete data sets is not a trivial one. This becomes more complicated when attempting to extract weak anomalous 
signals for de novo structure determination (Liu et al., 2012; Dauter, 2019). In addition, microcrystals are affected by environmental changes and their unit cells may have large variations. To rationally treat unit-cell variations, radiation damage and incomplete data in microcrystals, we and others have developed data assembly workflows (Guo et al., 2018, 2019; Yamashita et al., 2018; Basu et al., 2019; Cianci et al., 2019). With our workflow, we were able to assemble anomalous diffraction data from about 1200 native microcrystals and used the data for a successful single-wavelength anomalous diffraction analysis (Guo et al., 2019).

Here we describe the implementation of our microcrystal data assembly workflow in a Python program called $P y M D A$. $P y M D A$ allows for processing individual microcrystal data sets as progressive wedges to address radiation damage and allows for robust extraction of diffraction signals including weak anomalous signals through the implementation of unitcell-based classification and an iterative outlier rejection strategy. PyMDA may be used routinely to process microcrystal data sets to produce one or more assembled data sets for structural analysis.

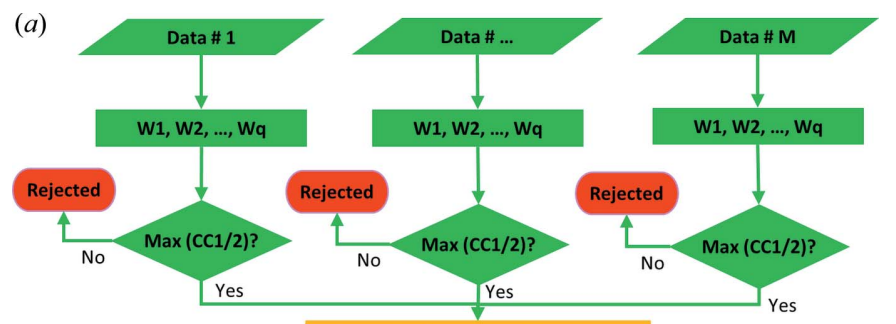

(b)

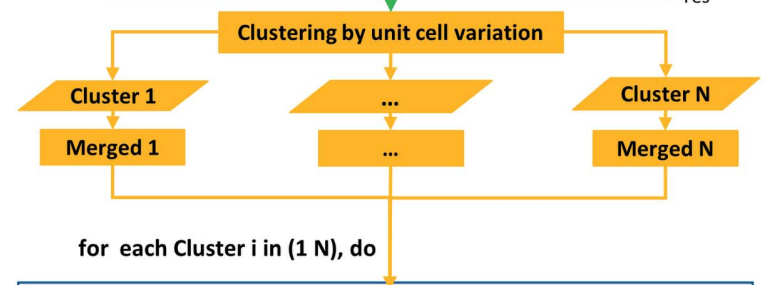

(c)

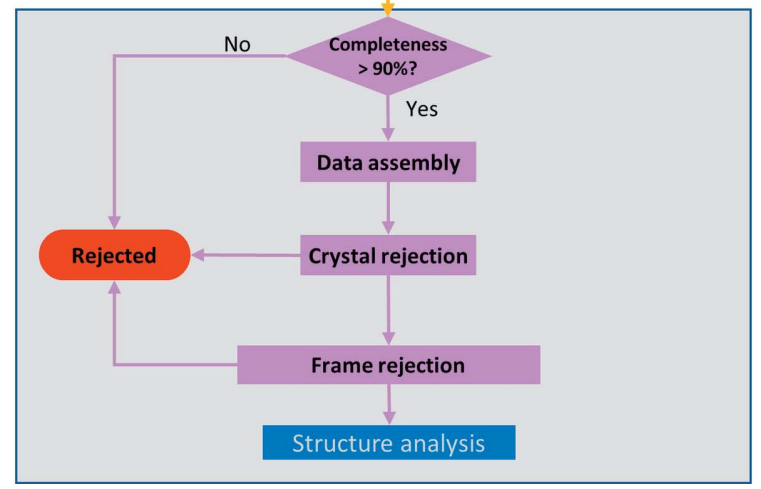

Figure 1

Multi-step data assembly workflow. (a) Progressive processing of singlecrystal data sets as accumulative wedges. $(b)$ Classification based on unitcell variations. (c) Data assembly for each cluster that qualified (completeness $>90 \%$ ). The data assembly procedure optimizes data quality by iterative crystal and frame rejections. PyMDA produces $N$ optimized data sets, each corresponding to a different set of unit-cell parameters.

\section{Implementation}

\subsection{Overall workflow}

The overall workflow of our microcrystal data assembly contains three main steps and is outlined schematically in Fig. 1. The first step is to process each of $M$ single-crystal data sets independently using DIALS (Waterman et al., 2016; Winter et al., 2018) and scale them using CCP4 programs POINTLESS and AIMLESS (Winn et al., 2011; Evans \& Murshudov, 2013) as $q$ progressive wedges [Fig. 1(a)]. Wedge $q$ includes all the preceding wedges 1 to $q-1$. The $\mathrm{CC}_{1 / 2}$ values reported in AIMLESS are used for selection of those wedges. Among these $q$ wedges, only the one that has the maximum $\mathrm{CC}_{1 / 2}$ is selected to form one of the $M$ single-crystal data sets. The second step is to classify these $M$ single-crystal data sets into $N$ classes using their unit-cell parameters [Fig. 1(b)]. Step 3 is to assemble data sets for each of the $N$ classes [Fig. 1(c)]. Those classes with merged data completeness greater than $90 \%$ are subject to iterative crystal and frame rejection. The quality of the assembled data is evaluated using data quality indicators: $\mathrm{CC}_{1 / 2}$ for high-resolution (diffraction limit) data and DelAnom for anomalous signals. Both $\mathrm{CC}_{1 / 2}$ and DelAnom are reported in AIMLESS. The assembled data sets may then be used directly for structure analysis including $d e$ novo phase determination.

\subsection{Python implementation}

PyMDA is implemented in Python 2.7 and requires the libraries of NumPy, SciPy and Matplotlib. In addition, PyMDA utilizes three external programs, DIALS, POINTLESS and AIMLESS, for single-crystal data processing and subsequent assembly. The program DIALS is used for indexing and integration; POINTLESS is used for data combination and re-indexing if necessary; and AIMLESS is used for scaling and merging including iterative crystal and frame rejections. The latest versions of DIALS, POINTLESS and AIMLESS should be used.

PyMDA takes input hdf5 format data collected from modern pixel array detectors such as the EIGER 16M and 9M (Casanas et al., 2016). The outputs for PyMDA are a series of assembled data sets that have been converted to structurefactor amplitudes with an added column for $R$ free flags. PyMDA uses the usual CCP4 programs CTRUNCATE, MTZ2VARIOUS and FREERFLAG to convert intensities and to add $R$ free flags.

\subsection{Single-crystal data processing}

Because of radiation damage, each of the single-crystal data sets is typically processed as accumulative wedges using DIALS. PyMDA has a command-line option--run_dials to facilitate the use of DIALS for processing individual data sets. PyMDA runs DIALS tools successively from data import to data export. These tools are dials.import, dials.find_spots, dials.index, dials.refine, dials.integrate and dials.export (Waterman et al., 2016). The required input parameter for --run_dials is the directory path (--hdf5_path) that 
contains the master hdf5 data files. Optional parameters for --run_dials are space group (--spg), unit-cell dimensions (--unit_cell), resolution (--reso), the number of wedges (--wedges) and the number of processes to be used (--thread). PyMDA processes many data sets automatically. However, for unknown samples, it is recommended to process a few data sets first to identify the space group and unit-cell parameters. If accurate detector parameters are known, it might be worth fixing the detector (--fix detector). By default, PyMDA uses a single process, but using multiple processes is possible and preferable.

There are two resolution cutoffs used in processing singlecrystal data sets. One (--reso) is the resolution at which the data will be processed, and the other one (--reso_cchalf) is for generating $\mathrm{CC}_{1 / 2}$ statistics using AIMLESS. The crystal is damaged, which results in a loss of intensity, particularly at high resolution. To have more frames selected for data assembly, we suggest using a statistics resolution lower than the data processing resolution (--reso) (Guo et al., 2018). By default, the value of --reso_cchalf is the value of --reso plus 1.

For challenging single-crystal data sets where the diffraction is weak, we provide an optional optimization step (--opt) to optimize three spot-finding parameters, sigma_b, sigma_s and minimum spot size, used by dials.find_spots. With this optimization mode, PyMDA will run a grid search for these parameters for a maximum $I / \sigma(I)$. In general, we only optimize these parameters for a selected data set; however, if desired, the optimization can be performed for all data sets. Be aware that this mode will take much longer. With known spotfinding parameters, $P y M D A$ can use them directly through parameters--sigb,--sigs and--minspot.

Each single-crystal data set is processed under a different directory with the same name as the prefix of its respective hdf5 data file. After single-crystal data processing, a series of data sets in mtz format and AIMLESS log files are produced. These mtz files are then used for data assembly; and AIMLESS $\log$ files are used to extract $\mathrm{CC}_{1 / 2}$ values and unitcell parameters. Below is an example of processing hdf5 format data:

/path-to-pymda/pymda --run_dials --hdf5_path /root-path-containing-hdf5-data/ --wedges 10 --spg p2221 --thread 4

\subsection{Classification by unit-cell variations}

The data sets selected with the highest $\mathrm{CC}_{1 / 2}$ values are used for classification based on unit-cell variations. With the extracted $M$ sets of unit-cell parameters, classification is performed (Liu et al., 2012, 2013; Foadi et al., 2013) with the desired number of classes. Unit-cell classification can be performed through a combination of keywords --run_mda and - ucr $=\mathrm{N}$, where $N$ is the number of desired classes. PyMDA writes out a PDF file to store the dendrogram plot that may be visualized and used as a reference to rerun the classification with a different number of classes. After unit-cell classification, PyMDA splits the $M$ data sets into $N$ classes. Below is an example of running classification for ten classes based on unit-cell variations:

/path-to-pymda/pymda --run_mda --dataprefix prefix_of_hdf5--ucr 10

Two further classification options are provided. Option --single is to maximize the number of crystals in a single class. This option is useful to keep more crystals together for optimizing anomalous signals. The option --ward is to maximize the separation of classes and this option is useful to obtain more assembled data sets.

\subsection{Crystal rejection}

Each of the $N$ classes with data completeness higher than $90 \%$ may be used for crystal rejection with an option --rjxtal. By default, PyMDA produces $N$ assembled data sets without crystal rejection. The goal of the crystal rejection step is to exclude single-crystal data sets that are not compatible with the merged one within each of the $N$ classes. To perform crystal rejection, PyMDA uses the per-frame smoothed $R_{\text {merge }}$ (SmRmerge) values extracted from AIMLESS. Prior to each iteration of crystal rejection, PyMDA computes the average of SmRmerge values 〈SmRmerge〉 for each crystal, sorts per-crystal 〈SmRmerge〉 and then rejects the specified number of crystals (defined by --xtal_step) with the highest $\langle$ SmRmerge $\rangle$ values. The iteration continues until the number of crystals is equal to or less than the number defined by --xtal_step. Below is an example of running crystal rejection with a rejection of ten crystals for each iteration:

/path-to-pymda/pymda --run_mda --dataprefix prefix_of_hdf5--ucr $10--r j x t a l--x t a l \_s t e p 10$

\subsection{Diffraction frame rejection}

Following each iteration of crystal rejection, $P y M D A$ can perform the rejection of those frames (--rjframe) that suffer too severely from radiation damage. Because of natural variations among microcrystals, there is no uniform rejection criterion suitable for all microcrystals. Therefore, PyMDA adopts a user-defined grid-search procedure for frame rejection (Guo et al., 2018). Frame rejection is optional and may be performed after each iteration of crystal rejection for each of the $N$ classes that qualified (e.g. with their data completeness higher than $90 \%$ ).

By defining the sequence of decay values, frame rejection is performed under different radiation-damage conditions to optimize the overall data quality. For each single-crystal data set, PyMDA identifies the frame number with the lowest SmRmerge and stores it as $\min$ (SmRmerge). The frame rejection cutoff is then defined as rjframe = $[\mathrm{min}(\mathrm{SmRmerge}) \times(1+$ decay $)]$, where decay is defined as each of the values in a sequence through an option --decay. The default sequence is '5.0 3.02 .01 .0 '. With the default 
sequence, $P y M D A$ rejects frames that have rjframe values 6,4 , 3 and 2 times $\min ($ SmRmerge). Four data sets are then produced, each corresponding to a different decay value. Below is an example of running frame rejection after each iteration of crystal rejection:

/path-to-pymda/pymda --run_mda --dataprefix prefix_of_hdf5 --ucr 10 --rjxtal --xtal_steps 10 --rjerame --decay "3.02.01.0"

After crystal and frame rejection, $P y M D A$ outputs multiple data sets and the associated AIMLESS log files, each derived from using different numbers of crystals and frames. It is suggested these data sets are sorted on the basis of different statistics reported in their AIMLESS log files. For a highresolution structure analysis that does not require anomalous signals, the data set with maximum $\mathrm{CC}_{1 / 2}$ is suitable. For $d e$ novo structure determination that uses anomalous signals, the data set with maximum DelAnom may be used.

\section{Discussion}

\subsection{Microcrystal data collection and processing}

$P y M D A$ assembles partial microcrystal diffraction data sets that are collected as small rotation wedges into complete crystallographic data sets. There is no special requirement for how many frames are examined by $P y M D A$. In developing $\operatorname{Py} M D A$, we anticipate ten to 100 frames per microcrystal with a rotation angle of 0.1 to $0.3^{\circ}$ per frame (1-30 of data per microcrystal). By processing single-crystal data sets as accumulative wedges, radiation-damaged frames should not compromise the overall data quality. Seriously damaged frames are rejected at the single-crystal data processing stage. One can use--reso_cchalf to fine tune the control of how many frames are to be rejected from data assembly. A smaller value of --reso_cchalf (higher resolution) results in more rejected frames.

The development of the current version of $P y M D A$ was based on the use of hdf5 format data created by EIGER detectors. PyMDA uses the master hdf5 names to create directories to execute parallel processing. It is a requirement that all hdf5 master files are under the same root directory and have the same prefix. This is the case for data collected at NSLS-II AMX and FMX beamlines and other macromolecular crystallography beamlines using EIGER detectors. Nevertheless, these master hdf5 files can be in different locations as long as they are under the same root directory defined by -- hdf5-path.

\subsection{Resolution cutoff}

PyMDA provides two resolution cutoffs for single-crystal data processing and assembly. --reso is used for indexing and integration and final assembly. --reso_cchalf is used for calculation of $\mathrm{CC}_{1 / 2}$ values of single-crystal data wedges. In the single-crystal data processing stage (--run_dials), it is suggested one uses the highest resolution by visual inspection of diffraction images during or after data collection. In the assembly stage (--run_mda), different resolution cutoffs may be used. For example, if a high-resolution data set is desirable, that resolution may be used for data assembly. All crystal and frame rejections will then be optimized against that resolution. Below is an example of running frame rejection after each iteration of crystal rejection at a resolution of $2.5 \AA$ :

/path-to-pymda/pymda --run_mda --dataprefix prefix_of_hdf5--ucr 10 --rjxtal--xtal_step 10 - -reso 2.5

If anomalous signals are to be optimized, we suggest using a lower resolution to enhance anomalous signals by increasing data redundancy. Because --run_dials and --run_mda can be performed successively, using two different resolutions through --reso can be done by running PyMDA twice: once at the highest resolution and once at a lower resolution (for optimized anomalous signals). Both data sets may then be used for structure refinement and phasing. For example, if the high-resolution data set is at $2.5 \AA$, below is an example of running $P y M D A$ to optimize anomalous signals at a lower resolution of $3.5 \AA$ :

/path-to-pymda/pymda --run_mda --dataprefix prefix_of_hdf5--ucr 10 --rjxtal--xtal_step 10 -reso 3.5

\subsection{Program limitations}

The current version of $P y M D A$ has limitations. Firstly, PyMDA can only process rotation data with the hdf5 data format. Support for cbf format will be provided in a future version. Secondly, the unit-cell classification is not fully automated. We suggest checking the output dendrogram plot to define the number of classes. Because of structural changes, manipulation or poor evaluation, microcrystals might have a wide spread of unit-cell parameters. One may need to manually remove certain single-crystal data sets that have unrealistic unit cells (e.g. by deleting their directories) before the fully automated data assembly can be run robustly. This can be done by checking the file check. txt. This file contains the directory names, the unit-cell parameters and their corresponding $\mathrm{CC}_{1 / 2}$ values for each of the single-crystal data sets. It would be useful to manually delete these extreme data sets with quite different unit-cell dimensions. These individual data sets may also be identified by running unit-cell classification with the --single option.

\section{Concluding remarks}

Microdiffraction data sets from microcrystals are difficult to process due to radiation damage, incompleteness and large unit-cell variations. Here we have implemented a Python program $P y M D A$ for microcrystal data assembly. $P y M D A$ assembles optimized data suitable for high-resolution structure analysis and de novo structure determination. PyMDA 
can not only assemble diffraction data from microcrystals, but also assemble data sets from larger crystals to improve data quality. $P y M D A$ is available from https://github.com/qun-liu/ pymda.

\section{Funding information}

This work was supported in part by Brookhaven National Laboratory LDRD 17-023, NIH grants GM107462 and P30GM133893, and DOE-BER grant KP1607010. LT was supported by the US Department of Energy, Office of Science, Office of Workforce Development for Teachers and Scientists (WDTS), under the Science Undergraduate Laboratory Internships Program (SULI).

\section{References}

Basu, S., Olieric, V., Leonarski, F., Matsugaki, N., Kawano, Y., Takashi, T., Huang, C.-Y., Yamada, Y., Vera, L., Olieric, N., Basquin, J., Wojdyla, J. A., Bunk, O., Diederichs, K., Yamamoto, M. \& Wang, M. (2019). IUCrJ, 6, 373-386.

Casanas, A., Warshamanage, R., Finke, A. D., Panepucci, E., Olieric, V., Nöll, A., Tampé, R., Brandstetter, S., Förster, A., Mueller, M., Schulze-Briese, C., Bunk, O. \& Wang, M. (2016). Acta Cryst. D72, 1036-1048.

Cianci, M., Nanao, M. \& Schneider, T. R. (2019). Acta Cryst. D75, 192-199.

Coquelle, N., Brewster, A. S., Kapp, U., Shilova, A., Weinhausen, B., Burghammer, M. \& Colletier, J.-P. (2015). Acta Cryst. D71, 11841196.

Dauter, Z. (2019). IUCrJ, 6, 503-504.

Diederichs, K. \& Wang, M. (2017). Methods Mol. Biol. 1607, 239-272.

Evans, G., Axford, D., Waterman, D. \& Owen, R. L. (2011). Crystallogr. Rev. 17, 105-142.

Evans, P. R. \& Murshudov, G. N. (2013). Acta Cryst. D69, 1204-1214.

Flot, D., Mairs, T., Giraud, T., Guijarro, M., Lesourd, M., Rey, V., van Brussel, D., Morawe, C., Borel, C., Hignette, O., Chavanne, J., Nurizzo, D., McSweeney, S. \& Mitchell, E. (2010). J. Synchrotron Rad. 17, 107-118.

Foadi, J., Aller, P., Alguel, Y., Cameron, A., Axford, D., Owen, R. L., Armour, W., Waterman, D. G., Iwata, S. \& Evans, G. (2013). Acta Cryst. D69, 1617-1632.

Fuchs, M. R., Bhogadi, D. K., Jakoncic, J., Myers, S., Sweet, R. M., Berman, L. E., Skinner, J., Idir, M., Chubar, O., McSweeney, S. \& Schneider, D. K. (2016). AIP Conf. Proc. 1741, 0300026.

Gao, Y., Xu, W., Shi, W., Soares, A., Jakoncic, J., Myers, S., Martins, B., Skinner, J., Liu, Q., Bernstein, H., McSweeney, S., Nazaretski, E. \& Fuchs, M. R. (2018). J. Synchrotron Rad. 25, 1362-1370.
Garman, E. F. \& Weik, M. (2017). J. Synchrotron Rad. 24, 1-6.

Gati, C., Bourenkov, G., Klinge, M., Rehders, D., Stellato, F., Oberthür, D., Yefanov, O., Sommer, B. P., Mogk, S., Duszenko, M., Betzel, C., Schneider, T. R., Chapman, H. N. \& Redecke, L. (2014). IUCrJ, 1, 87-94.

Guo, G., Fuchs, M. R., Shi, W., Skinner, J., Berman, E., Ogata, C. M., Hendrickson, W. A., McSweeney, S. \& Liu, Q. (2018). IUCrJ, 5, 238-246.

Guo, G., Zhu, P., Fuchs, M. R., Shi, W., Andi, B., Gao, Y., Hendrickson, W. A., McSweeney, S. \& Liu, Q. (2019). IUCrJ, 6, 532-542.

Holton, J. M. \& Frankel, K. A. (2010). Acta Cryst. D66, 393-408.

Huang, C.-Y., Olieric, V., Howe, N., Warshamanage, R., Weinert, T., Panepucci, E., Vogeley, L., Basu, S., Diederichs, K., Caffrey, M. \& Wang, M. (2018). Commun. Biol. 1, 124.

Liu, Q., Dahmane, T., Zhang, Z., Assur, Z., Brasch, J., Shapiro, L., Mancia, F. \& Hendrickson, W. A. (2012). Science, 336, 1033-1037.

Liu, Q., Liu, Q. \& Hendrickson, W. A. (2013). Acta Cryst. D69, 13141332.

Owen, R. L., Axford, D., Sherrell, D. A., Kuo, A., Ernst, O. P., Schulz, E. C., Miller, R. J. D. \& Mueller-Werkmeister, H. M. (2017). Acta Cryst. D73, 373-378.

Sanishvili, R. \& Fischetti, R. F. (2017). Methods Mol. Biol. 1607, 219238.

Schlichting, I. (2015). IUCrJ, 2, 246-255.

Smith, J. L., Fischetti, R. F. \& Yamamoto, M. (2012). Curr. Opin. Struct. Biol. 22, 602-612.

Spence, J. C. H. (2017). IUCrJ, 4, 322-339.

Warren, A. J., Axford, D. \& Owen, R. L. (2019). J. Synchrotron Rad. 26, 991-997.

Waterman, D. G., Winter, G., Gildea, R. J., Parkhurst, J. M., Brewster, A. S., Sauter, N. K. \& Evans, G. (2016). Acta Cryst. D72, 558-575.

Winn, M. D., Ballard, C. C., Cowtan, K. D., Dodson, E. J., Emsley, P., Evans, P. R., Keegan, R. M., Krissinel, E. B., Leslie, A. G. W., McCoy, A., McNicholas, S. J., Murshudov, G. N., Pannu, N. S., Potterton, E. A., Powell, H. R., Read, R. J., Vagin, A. \& Wilson, K. S. (2011). Acta Cryst. D67, 235-242.

Winter, G., Waterman, D. G., Parkhurst, J. M., Brewster, A. S., Gildea, R. J., Gerstel, M., Fuentes-Montero, L., Vollmar, M., MichelsClark, T., Young, I. D., Sauter, N. K. \& Evans, G. (2018). Acta Cryst. D74, 85-97.

Yamamoto, M., Hirata, K., Yamashita, K., Hasegawa, K., Ueno, G., Ago, H. \& Kumasaka, T. (2017). IUCrJ, 4, 529-539.

Yamashita, K., Hirata, K. \& Yamamoto, M. (2018). Acta Cryst. D74, 441-449.

Zander, U., Bourenkov, G., Popov, A. N., de Sanctis, D., Svensson, O., McCarthy, A. A., Round, E., Gordeliy, V., Mueller-Dieckmann, C. \& Leonard, G. A. (2015). Acta Cryst. D71, 2328-2343.

Zeldin, O. B., Brockhauser, S., Bremridge, J., Holton, J. M. \& Garman, E. F. (2013). Proc. Natl Acad. Sci. USA, 110, 20551-20556. 